\title{
Sunspot Time Series: Passive and Active Intervals
}

\author{
S. Zięba $\cdot$ Z. Nieckarz
}

Received: 26 September 2013 / Accepted: 7 February 2014 / Published online: 26 February 2014

(C) The Author(s) 2014. This article is published with open access at Springerlink.com

\begin{abstract}
Solar activity slowly and irregularly decreases from the first spotless day (FSD) in the declining phase of the old sunspot cycle and systematically, but also in an irregular way, increases to the new cycle maximum after the last spotless day (LSD). The time interval between the first and the last spotless day can be called the passive interval (PI), while the time interval from the last spotless day to the first one after the new cycle maximum is the related active interval (AI). Minima of solar cycles are inside PIs, while maxima are inside AIs. In this article, we study the properties of passive and active intervals to determine the relation between them. We have found that some properties of PIs, and related AIs, differ significantly between two group of solar cycles; this has allowed us to classify Cycles $8-15$ as passive cycles, and Cycles 17-23 as active ones. We conclude that the solar activity in the PI declining phase (a descending phase of the previous cycle) determines the strength of the approaching maximum in the case of active cycles, while the activity of the PI rising phase (a phase of the ongoing cycle early growth) determines the strength of passive cycles. This can have implications for solar dynamo models. Our approach indicates the important role of solar activity during the declining and the rising phases of the solar-cycle minimum.
\end{abstract}

Keywords Spotless days $\cdot$ Solar phase tracking $\cdot$ Waldmeier effect $\cdot$ Prediction

\section{Introduction}

In a recently published paper (Zięba and Nieckarz, 2012), we have shown that the relations based on the position of the longest spotless segment (LSS, the longest sequence of consecutive days when no spots were observed) with respect to locations of some characteristic

\footnotetext{
S. Zięba (凶)

Astronomical Observatory, Jagiellonian University, Orla 171, 30-244, Kraków, Poland e-mail: stanzieba712@gmail.com
}

\section{Z. Nieckarz}

Institute of Physics, Jagiellonian University, Reymonta 4, 30-059, Kraków, Poland

e-mail: zenon.nieckarz@uj.edu.pl 
extreme points in the daily sunspot numbers are statistically significant and have a predictive value. This indicates that the passive interval (PI) as well as the location and length of the LSS can be useful for studying of the physical processes responsible for the solar variability, especially since their values do not result from any smoothing procedure but are really observed.

In this article, we examine relationships between various parameters characterizing the passive and active intervals whose definition was given by Zięba et al. (2006). The active interval (AI) complements the passive interval in the sense that its covers also the time interval between the border spotless days but includes the cycle maximum. The proposed decomposition of sunspot time series into passive and active intervals is unambiguous. PI and related AI carry the same number, which is assigned from the number of the cycle of which minimum and maximum are inside of these intervals. Each passive interval and the active interval occurring after it form the ordered pair PI-AI. As the classically understood solar cycle and the pair PI-AI with the same number include the same maximum phase of solar cycle we use the name cycle also for the ordered pair PI-AI.

The basis for our research was and is the daily international sunspot number (ISN) series provided by the Solar Influences Data Center (SIDC, http://sidc.oma.be/) of the Royal Observatory of Belgium. Until 1980 the ISN, better known as the Wolf or Zürich number, was compiled by the Swiss Federal Observatory. In this work we use the daily ISN series covering the period between January 1830 and June 2012. This period includes the decline phase of Cycle 7, Cycles 8-23 and the initial rise of Cycle 24. Cycles 7-9 include the era of Schwabe's records with a large number of days without observations (Wilson, 1998). Cycles 10, 11, and the rise of Cycle 12 belong to Wolf's era (years $1848-1882$ ), while those of Cycles 13-21 belong to the Zürich era. Since 1981, when the International Astronomical Union World Data Center for sunspot numbers was transferred from the Zürich Observatory to Brussels, a new approach for calculation of the sunspot number has been established (Clette et al., 2007). The intrinsic nature and accuracy for four main eras of sunspot number observations are different in the ISN time series and this is the reason for treating the daily ISN series with care. However, we have shown in Zięba and Nieckarz (2012) that differences arising from heterogeneous ISN data among Cycles 8-23 can be neglected.

This article is organized as follows. In Section 2 we describe characteristic points of the daily ISN series and define various time intervals whose lengths and amplitudes are studied in Sections 3 and 4. Next, in Section 5 we discuss relations between lengths (periods) and amplitudes (strength) of the intervals considered, including the Waldmeier effect. Some predictions for Cycle 24 are given in Section 6, and Section 7 comprises a summary and a discussion.

\section{Definition of Characteristic Points and Time Intervals}

The passive interval (PI) is defined as the time interval (denoted ' 00 ') from the first spotless day (FSD) after an old cycle maximum to the last spotless day (LSD) before the next new cycle maximum (Zięba et al., 2006; Zięba and Nieckarz, 2012). All spotless days occur within the passive intervals. For each passive interval we have a minimum of activity (cycle minimum) and the occurrence of the longest spotless segment (LSS). We accept the center of LSS as the distinctive point of the PI.

The idea of using spotless days to find the minimum of activity was suggested by Waldmeier (1961) and McKinnon (1987). Wilson (1995) proposed to use the first spotless day as 

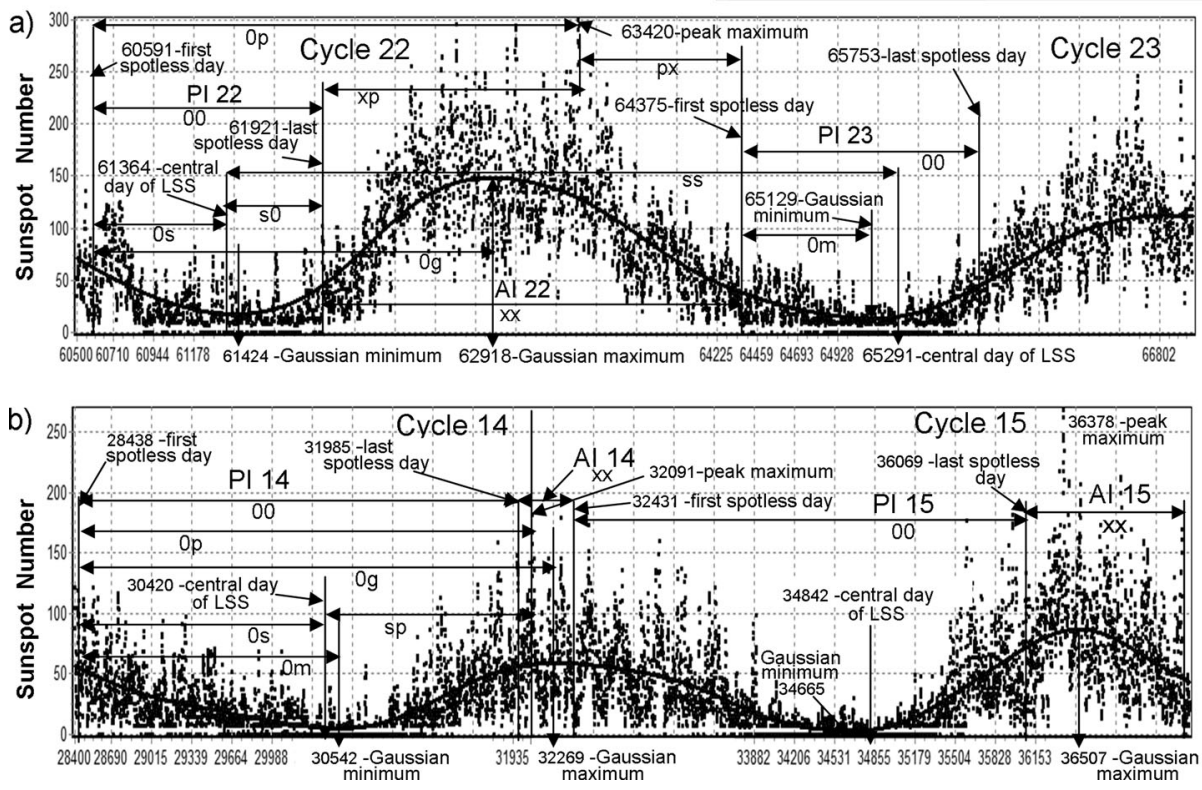

Figure 1 The daily values of ISN drawn for passive (PI) and active (AI) time intervals of Cycles 14, 15, 22, and 23. The black curve represents the data smoothed with the Gaussian filter of full width at half maximum (FWHM) of 810 days. LSS stands for the longest spotless segment; it is the longest sequence of consecutive days when no spots were observed. The positions of the characteristic extreme points near sunspot minimum and maxima are denoted together with various time intervals used in this article and defined in Table 1 . The numbers are enumerated from 1 January 1818 (for dates see Table 2).

a predictor for the sunspot minimum. The possible connections of spotless days with the timing and length of the solar cycle were more accurately examined by Wilson and Hathaway (2005, 2006, 2007).

The time interval (denoted 'xx'), between the last spotless day before the upcoming new cycle maximum and the first spotless day after it, includes all the days when sunspots are observed and we called it the active interval (AI). The day with the highest sunspot number (RP, the day with the maximum number of sunspots in a given cycle) is inside AI and we consider its position as an AI distinctive point.

Figure 1(a) presents the PI and AI for Cycles 22 and 23, while Figure 1(b) shows the same for Cycles 14 and 15. Various time intervals used in this article are also marked. They are defined in Table 1, while Table 2 lists the positions of the distinctive points within each solar cycle and the calculated lengths of intervals. Comparison of Cycle 22 (see Figure 1(a)) with Cycle 14 (see Figure 1(b)) shows the clear difference between the passive and active intervals related to these cycles. Cycle 22 is untypical because inside its AI there are three peaks, almost equal in size: 296, 295, and 300. The active interval of Cycle 14 is the shortest one $(\mathrm{xx}=445)$ from all the cycles.

The definition of PI and AI, as well as the location of the LSS and the peak maximum, is based on the precisely determined observables. For comparative purposes we also distinguish the days having the minimal (RGm) and maximal (RG) sunspot number after smoothing the ISN time series using an 810-day Gaussian filter. The Gaussian filter, having a full width at half maximum (FWHM) equal to 810 days, effectively removes short-term variations of solar activity on time scales of about two years that can produce double peaked 
Table 1 Definitions of the intervals and the time distances used in this article.

\begin{tabular}{|c|c|c|c|c|c|}
\hline \multirow{3}{*}{$\begin{array}{l}\text { Interval } \\
\text { (distance) }\end{array}$} & \multirow[t]{3}{*}{ Definition } & \multicolumn{2}{|l|}{ Cycle } & \multicolumn{2}{|c|}{ All cycle } \\
\hline & & 14 & 22 & Mean & sem $^{1}$ \\
\hline & & \multicolumn{4}{|l|}{ [days] } \\
\hline $00^{2}$ & $\begin{array}{l}\text { Passive interval (PI), the time interval covering all the } \\
\text { days from the first spotless day (FSD) after a cycle } \\
\text { maximum to the last spotless day (LSD) before the } \\
\text { next cycle maximum along with them }\end{array}$ & 3548 & 1331 & 2334 & 216 \\
\hline $0 \mathrm{~s}$ & $\begin{array}{l}\text { Declining segment of the PI, the time interval from the } \\
\text { FSD after a cycle maximum to the central day of the } \\
\text { LSS }\end{array}$ & 1983 & 774 & 1512 & 131 \\
\hline s0 & $\begin{array}{l}\text { Rising segment of the PI, the time interval from the } \\
\text { central day of the LSS to the LSD before the next } \\
\text { cycle maximum }\end{array}$ & 1565 & 557 & 822 & 100 \\
\hline $0 \mathrm{~m}$ & $\begin{array}{l}\text { The time distance from the FSD after a cycle } \\
\text { maximum to the day of minimum given by the } \\
\text { 810-day Gaussian filter (Gaussian minimum, Gm) }\end{array}$ & 2105 & 834 & 1404 & 138 \\
\hline $0 p$ & $\begin{array}{l}\text { The time distance from the FSD after a cycle } \\
\text { maximum to the day with the maximal daily sunspot } \\
\text { number (peak maximum, Px) inside the next cycle } \\
\text { maximum }\end{array}$ & 3653 & 2829 & 2919 & 164 \\
\hline $0 \mathrm{~g}$ & $\begin{array}{l}\text { The time distance from the FSD after a cycle } \\
\text { maximum to the day of maximum given by the } \\
\text { 810-day Gaussian filter (Gaussian maximum, Gx) } \\
\text { inside the next cycle maximum }\end{array}$ & 3831 & 2327 & 3020 & 153 \\
\hline $\mathrm{sp}$ & $\begin{array}{l}\text { The time distance from the central day of the LSS to } \\
\text { the successive Px }\end{array}$ & 1671 & 2056 & 1407 & 74 \\
\hline $\mathrm{xx}$ & $\begin{array}{l}\text { Active interval (AI), the time interval covering all the } \\
\text { days from the LSD after a cycle minimum to the FSD } \\
\text { before the next cycle minimum without spotless days }\end{array}$ & 445 & 2453 & 1638 & 173 \\
\hline $\mathrm{xp}$ & $\begin{array}{l}\text { The time distance from the LSD before a cycle } \\
\text { maximum to the Px }\end{array}$ & 106 & 1499 & 585 & 97 \\
\hline $\mathrm{px}$ & $\begin{array}{l}\text { The time distance from the Px to the FSD before the } \\
\text { next cycle minimum }\end{array}$ & 339 & 954 & 1052 & 109 \\
\hline ss & $\begin{array}{l}\text { The time distance between the central days of two } \\
\text { successive LSS }\end{array}$ & 4422 & 3927 & 4002 & 86 \\
\hline
\end{tabular}

1 sem, the standard error of the mean.

2 Intervals ' 00 ', ' $\mathrm{xx}$ ', and two others similar to ' $0 \mathrm{~m}$ ' and ' $0 \mathrm{~g}$ ' were considered by Wilson and Hathaway in 2005 in a NASA Technical Report. They used a smoothed (12 month moving average) monthly mean sunspot number for calculation their time parameters.

maxima. The size of this filter is similar to the 24-month Gaussian filter used when monthly averaged sunspot numbers are considered (Hathaway, Wilson, and Reichmann, 1999; Hathaway, 2010).

Differences between the position of the LSS and the Gaussian minimum as well as between the peak maximum and the Gaussian one are small. Figure 2 depicts their locations measured with respect to the FSD. The correlation coefficients between ' $0 \mathrm{~s}$ ' and ' $0 \mathrm{~m}$ ' $(r=0.965)$ as well as between '0p' and '0g' $(r=0.949)$ are very high. In Figure 2(b), Cycle 22 clearly stands out of the relation we have found; this is probably due to the presence of three almost equal peaks (see Figure 1(a)). When the relation is calculated without in- 


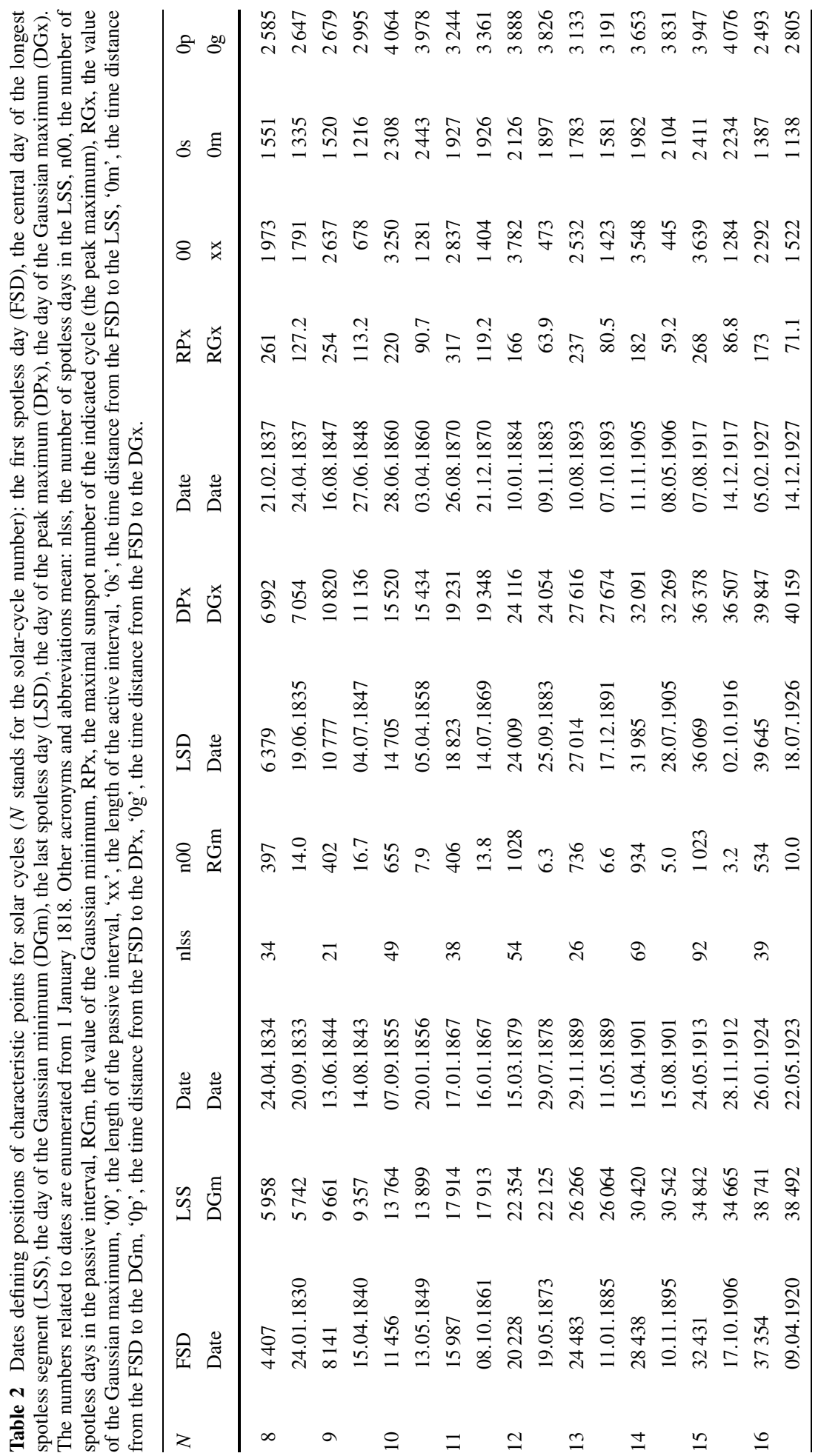




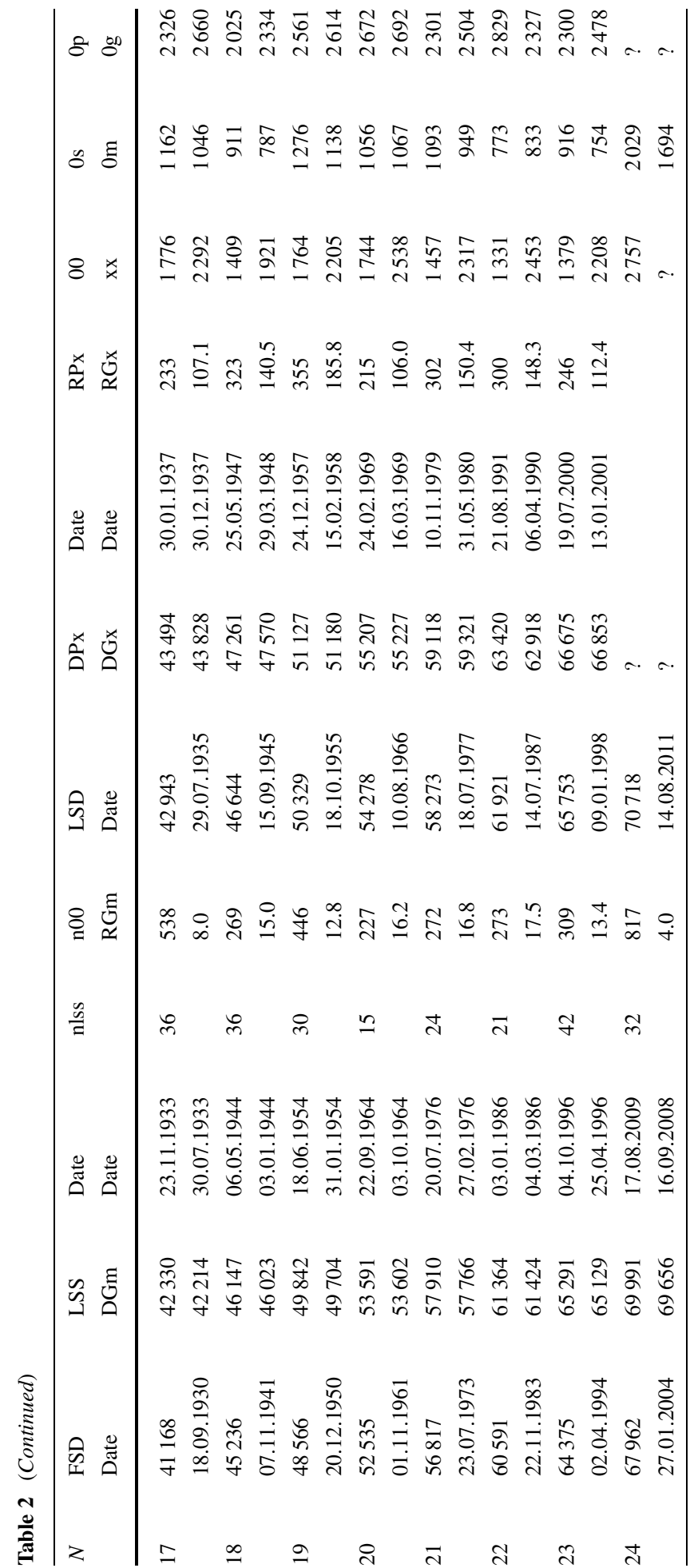



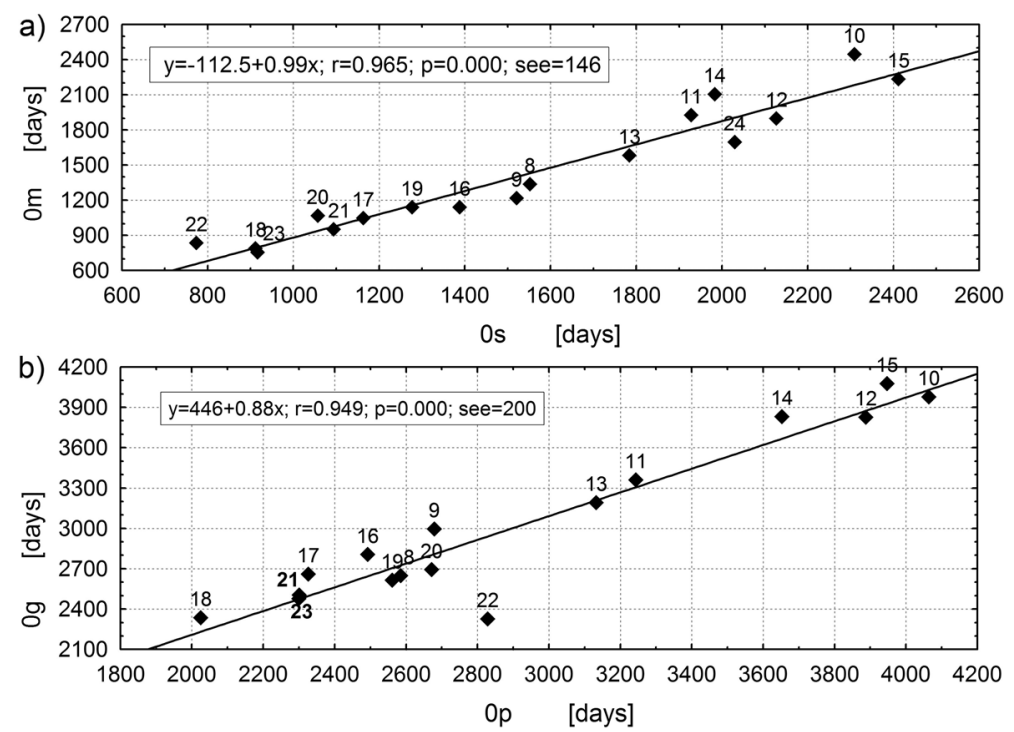

Figure 2 (a) Scatterplot of time distances '0m' (positions of the Gaussian minimum) versus '0s' (positions of the LSS). (b) Similar to (a) for '0g' (positions of the Gaussian maximum) versus 'Op' (positions of the peak maximum) for the indicated cycles. The equation of the best linear fit, the correlation coefficient $(r)$, the significance level $(p)$, and the standard error of the estimation (see) are also given.

cluding Cycle 22, then the correlation grows ( $r=0.98$ and see $=110$, where 'see' stands for standard error of the estimation). The mean and standard deviation ( $\mu$ and $\sigma$ in days) for the differences '0s', '0m' and '0p', '0g' are (122 and 141) and (-101 and 208), respectively. The high correlations and small differences between ' $0 \mathrm{~s}$ ' and ' $0 \mathrm{~m}$ ' and between ' $0 \mathrm{p}$ ' and ' $0 \mathrm{~g}$ ' confirm the treatment of the LSS and the peak maxima as observables describing minima and maxima of solar activity.

We suppose that the central day of the LSS represents better the time of real minimum, interpreted according to Cameron and Schüssler (2008) as the epoch when the sum of the activities of the old and the new cycle is minimal, than the Gaussian minimum. Various definitions of the cycle minimum were considered by Harvey and White (1999), who among others took into account the monthly averages of spotless days.

\section{Lengths of Intervals - Two Types of Solar Cycles}

Lengths of passive and active intervals vary from cycle to cycle, as is seen in Figure 3.

Passive intervals associated with Cycles $9-15$ ('00' $=3175 \pm 192$, the mean and the standard error of the mean are given) are clearly longer than the related active intervals ( $\mathrm{xx}^{\prime}=998 \pm 168$ ). The mean and the standard error of the ratio are ' $\mathrm{xx} / 00$ ' $=0.330 \pm$ 0.065 . In contrast to these cycles, Cycles $17-23$ have short passive intervals (' 00 ' $=1551 \pm$ 76 ) and long active intervals (' $\mathrm{xx}$ ' $=2276 \pm 75$ ) for which the mean and its standard error are ' $x x / 00$ ' $=1.485 \pm 0.079$ (see Figure 4). Despite the large differences between the ratios $\mathrm{xx} / 00$, the additions of passive to active intervals do not differ so much for these two types of solar cycles.

To check which parameters characterizing the passive and active intervals are significantly different in both types of cycles, we applied, because of the very small number of 


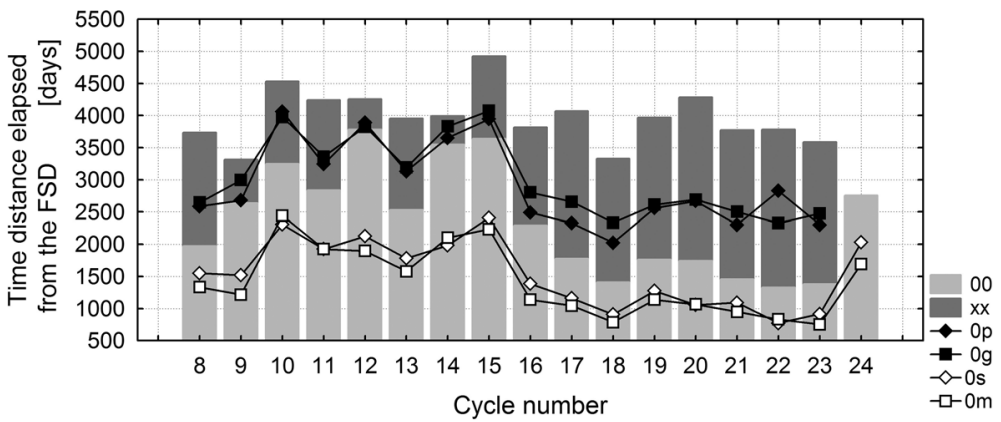

Figure 3 Cyclic variation of time distances measuring positions of the characteristic extreme points of the daily sunspot series with respect to the first spotless day (FSD) for Cycles 8-24. '0s', the position of the central day of the longest spotless segment, '0m', the position of the Gaussian minimum, '0p', the position of the peak maximum, ' $0 \mathrm{~g}$ ', the position of the Gaussian maximum. Vertical bars show lengths of passive ' 00 ' and active ' $x x$ ' intervals for Cycles $8-24$.

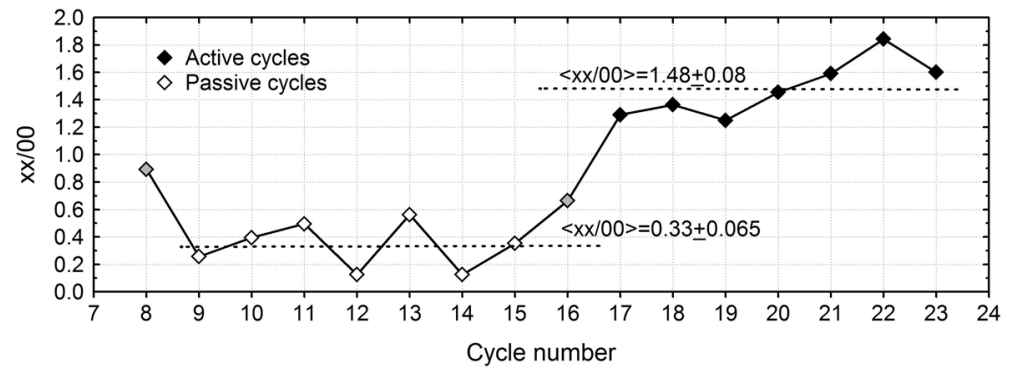

Figure 4 Scatterplot of the ratio xx/00 between lengths of active and passive intervals for Cycles $8-23$. The mean values and the standard errors of the means are shown for the two selected types of cycles.

cases ( 7 cases in each type), two statistical tests: Student's t-test and the Mann-Whitney U non-parametric test. The results are presented in Table 3.

The conclusions from a careful analysis of Table 3 are:

i) Lengths of cycles measured from one LSS to the next one (see row 9) and the time distance from the LSS minimum to the peak maximum are the same for both types of cycles (row 8).

ii) There are significant differences between both types of cycles in the lengths of their segments, such as '0s', 's0', 'xp', and 'px' (rows 2, 3, 5, 6).

iii) The AI mean sunspot numbers of Cycles 17-23 are sightly higher than those of Cycles $9-15$, but the differences are mainly in the 'xp' segments (rows 12,13).

iv) The PI mean sunspot numbers of Cycles 9-15 are significantly larger than those in the other type of cycles; this is mainly because of the relatively large sunspot numbers in their rising segments ' $\mathrm{s} 0$ ', along which a high variability of the sunspot numbers is also observed (rows 10, 11).

v) The number of the spotless days, the longest spotless segments and longest segments of days with spots in a row are significantly larger in the PI of Cycles 9- 15 (rows 14, 15, 16). 
Table 3 Results of statistical tests calculated for various parameters of the passive and active intervals.

\begin{tabular}{|c|c|c|c|c|c|c|c|c|}
\hline \multirow[t]{2}{*}{ No. } & \multirow[t]{2}{*}{ Parameter } & \multicolumn{2}{|c|}{ Cycles 9-15 } & \multicolumn{2}{|c|}{ Cycles $17-23$} & \multicolumn{3}{|c|}{$p$-value ${ }^{1}$} \\
\hline & & Mean & SD & Mean & SD & Test F & Test t & Test U \\
\hline 1 & Length of the PI ' 00 ' & 3175.0 & 507.5 & 1551.4 & 200.1 & 0.039 & 0.00000 & 0.0006 \\
\hline 2 & $\begin{array}{l}\text { Length of the PI declining segment } \\
\text { '0s' }\end{array}$ & 2009.1 & 306.1 & 1027.7 & 171.1 & 0.183 & 0.00001 & 0.0006 \\
\hline 3 & Length of the PI rising segment 's0' & 1165.9 & 340.6 & 523.7 & 106.0 & 0.012 & 0.00046 & 0.0006 \\
\hline 4 & Length of the AI ' $\mathrm{xx}$ ' & 998.3 & 445.6 & 2276.3 & 199.0 & 0.071 & 0.00002 & 0.0006 \\
\hline 5 & Length of the AI rising segment ' $x p$ ' & 341.4 & 287.8 & 880.1 & 308.9 & 0.868 & 0.00551 & 0.0070 \\
\hline 6 & $\begin{array}{l}\text { Length of the AI declining } \\
\text { segment 'px' }\end{array}$ & 656.9 & 278.6 & 1396.1 & 253.7 & 0.826 & 0.00022 & 0.0023 \\
\hline 7 & Ratio $\mathrm{xx} / 00$ & 0.330 & 0.171 & 1.485 & 0.209 & 0.637 & 0.00000 & 0.0006 \\
\hline 8 & $\begin{array}{l}\text { Time elapsed since the LSS to the } \\
\text { day of the peak maximum 'sp' }\end{array}$ & 1507.3 & 236.9 & 1403.9 & 332.8 & 0.428 & 0.516 & 0.383 \\
\hline 9 & $\begin{array}{l}\text { Time distance between two } \\
\text { subsequent LSS segments 'ss' }\end{array}$ & 4154.3 & 216.0 & 3951.6 & 422.0 & 0.128 & 0.280 & 0.209 \\
\hline 10 & Mean sunspot number of the PI & 30.86 & 6.82 & 22.07 & 4.95 & 0.455 & 0.01729 & 0.0379 \\
\hline 11 & $\begin{array}{l}\text { Mean sunspot number of ' } \mathrm{s} 0 \text { ' } \\
\text { segment in the PI }\end{array}$ & 30.39 & 11.29 & 18.13 & 2.87 & 0.004 & 0.01656 & 0.0262 \\
\hline 12 & Mean sunspot number of the AI & 85.64 & 23.20 & 106.15 & 20.77 & 0.795 & 0.10684 & 0.0973 \\
\hline 13 & $\begin{array}{l}\text { Mean sunspot number of 'xp' } \\
\text { segment in the AI }\end{array}$ & 82.37 & 14.26 & 105.48 & 27.56 & 0.134 & 0.07239 & 0.0728 \\
\hline 14 & Number of spotless days in the PI & 740.6 & 268.9 & 337.7 & 123.2 & 0.079 & 0.00362 & 0.0070 \\
\hline 15 & Length of the LSS & 49.86 & 24.84 & 29.14 & 9.60 & 0.036 & 0.062 & 0.0728 \\
\hline 16 & $\begin{array}{l}\text { Length of the longest segment of } \\
\text { days with spot in a row in the PI }\end{array}$ & 373.1 & 131.6 & 191.9 & 121.5 & 0.851 & 0.02011 & 0.0262 \\
\hline
\end{tabular}

$1 p$-value, the probability of the test statistic used for verifying the null hypothesis of tests: Test F, the F-test of equality of variances, Test $t$, the Student's t-test for the comparison of two means, Test U, the MannWhitney U non-parametric test for comparing two samples.

Large and statistically significant differences between various parameters of intervals associated with the two types of solar cycles lead us to assume that the spacing of days with small and large sunspot numbers are different. For Cycles $9-15$, the probability to find a day with a relatively large sunspot number outside of the active intervals is higher than in cycles of the other type (compare Figure 1(a) with Figure 1(b) and Figure 5). We think that this division is not random but reflects some physical reason, which would explain the observed features of the pair PI-AI in these groups. Further in this article we call Cycles 9-15 the passive cycles and Cycles 17-23 the active ones (see Figure 4).

\section{Amplitudes of Passive and Active Cycles}

Active and passive cycles differ largely not only in the previously discussed interval lengths, but also in other features. Besides the lengths, the amplitude is the second key characteristic of a cycle. We distinguish four various measures of a cycle amplitude which can be specified for any defined time segment ('ts'): the peak amplitude RPts, the highest sunspot number during 'ts', the mean amplitude RMts, the mean sunspot number calculated from days in the 'ts', the Gaussian maximum RGts, the maximal sunspot number in the 'ts' after smoothing 
Figure 5 Distribution of the days with spots in the three phases of a solar cycle: 'growth', the time interval from the longest spotless segment (LSS) minimum to the last spotless day (LSD), 'maximum', the time interval between the LSD and the first spotless day (FSD) comprising the peak maximum, 'decay', the time interval between the FSD and the next LSS minimum. (a) Days with the spot number greater than 0.25 of the peak maximum. (b) Days with the spot number greater than 0.5 of the peak maximum.

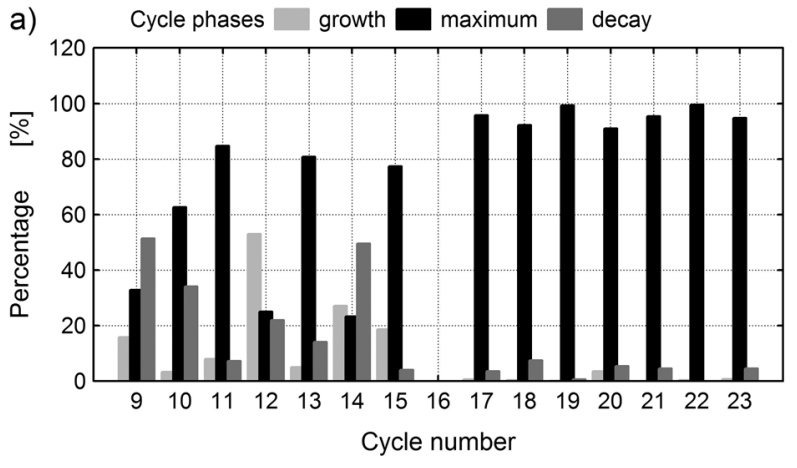

b) Cycle phases $\square$ growth $\square$ maximum $\square$ decay

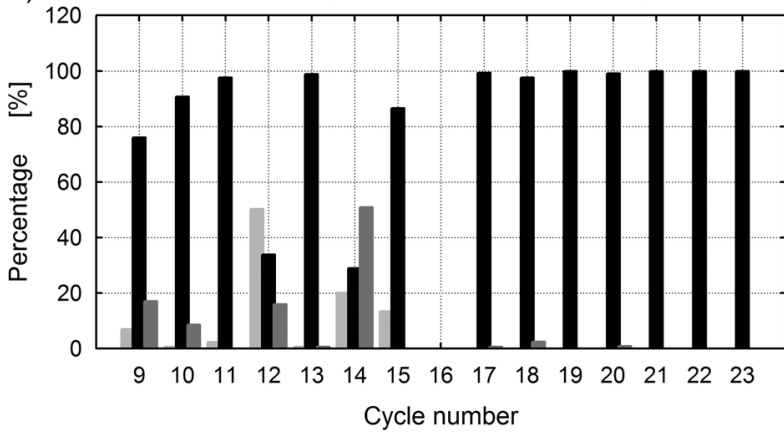

the ISN time series using the 810-day Gaussian filter, the integrated amplitude RIts, the sum of daily sunspot numbers in the 'ts'. We examine the following time segments: the passive interval ' 00 ', the declining segment of the passive interval ' 0 s', the rising segment of the passive interval 's0', the active interval ' $\mathrm{xx}$ ', the rising segment of the active interval 'xp' (from the beginning of the active interval to the day of peak maximum), and the declining segment of the active interval 'px'. Figure 6 shows the peak amplitudes which are observable values. There are three peak amplitudes linked with each active interval and two linked with each passive interval. This is a consequence of the clear division of each active and passive interval into two segments.

For the active interval, the day of the peak maximum is used as the split point, while for the passive interval, the central day of the LSS fulfills this role. The peak maxima in both segments of the passive interval are simple to define but for the active interval an additional rule is that the day corresponding to the maximum in each segment has to belong to a different solar rotation from the day of the split point.

For the active cycles the following characteristics can be noticed.

- All the three active interval peak amplitudes are apparently higher than peaks observed during passive intervals. Student's t-test gives the $p$-values (see the footnote in Table 3) smaller than 0.0006 for all the possible pairs of AI peak amplitudes versus PI peaks. Furthermore, we find that the non-parametric Wilcoxon $\mathrm{T}$ test (a signed-rank paired difference test) confirms these differences by giving a $p$-value of 0.018 for all the pairs.

- In Figure 6, the peaks of the PI declining segments of active cycles (red circles) are typically higher than the peaks of the rising segments (red triangles). The non-parametric Wilcoxon $\mathrm{T}$ test gives a $p$-value equal to 0.028 . 


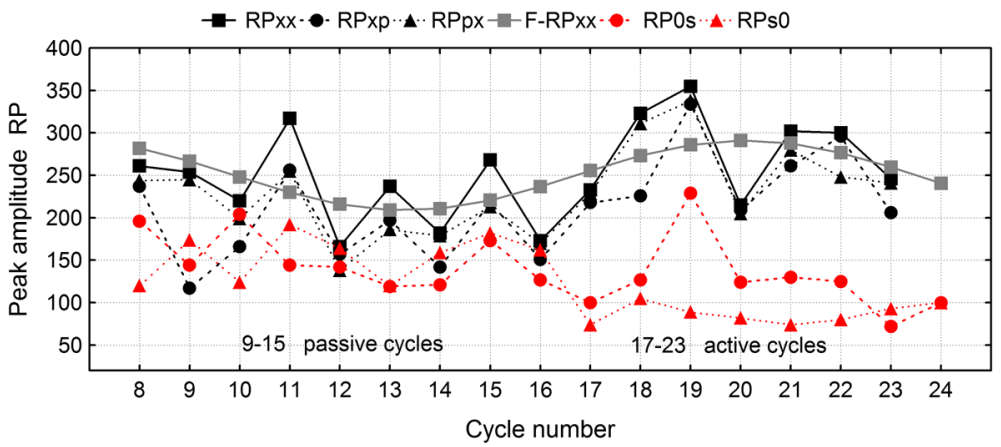

Figure 6 The scatterplots of the peak amplitude (RP) for the following time segments: the active interval ' $x x^{\prime}$ ' (RPxx), the rising segment of the active interval 'xp' (RPxp), the declining segment of the active interval 'px' (RPpx), the declining segment of the passive interval '0s' (RP0s), the rising segment of the passive interval 's0' (RPs0). The best sinusoidal fit (F-RPxx) for the active interval peak amplitudes is also shown. The black color is for active intervals and the red one for passive intervals.

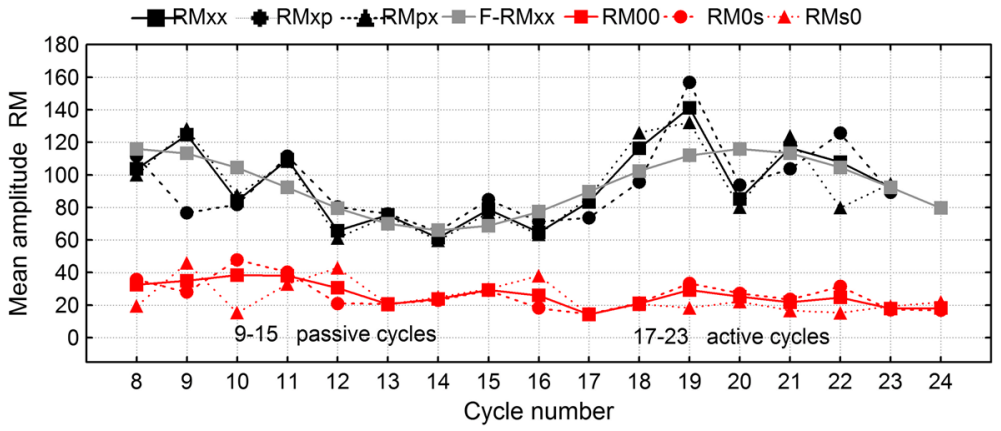

Figure 7 Similar to Figure 6, but in this case the scatterplots of the mean amplitude (RM) are shown. RM00 stands for the mean amplitude of the passive interval. The best sinusoidal fit (F-RMxx) for the active interval mean amplitudes is also shown.

- The peaks of PI rising segments of active cycles are significantly lower than the same one in the passive cycles. Student's t-test gives a $p$-value equal to 0.000027 while $p$-value of the Mann-Whitney U non-parametric test is 0.00058 .

The differences among peak amplitudes of the active and passive intervals for the passive cycles are still evident, but peaks of the AI rising segments (black circles) are not significantly higher than those characterizing the passive segments. Figures 7 to 9 are similar to Figure 6 but depict the other defined amplitudes.

In Figure 7, the curves of the mean amplitudes support the same results we infer from the curves in Figure 6. The statistical significance of the differences between the mean amplitudes of the active and passive intervals for all the cycles is evident. Results of the comparison between the mean amplitudes of the passive and active cycles calculated separately for the passive and active intervals are given in Table 3 (row 10 and 12).

Both peak and mean amplitudes of the active intervals (except for the even-odd cycle pair $22-23$ and, probably, 8-9) follow the Gnevyshev-Ohl rule, which states that in consecutive odd-even cycle pairs the odd cycle tends to be the stronger (Gnevyshev and Ohl, 1948; Kopecky, 1950). Also the curves of the Gaussian maximum amplitude (RGxx in Figure 8) 


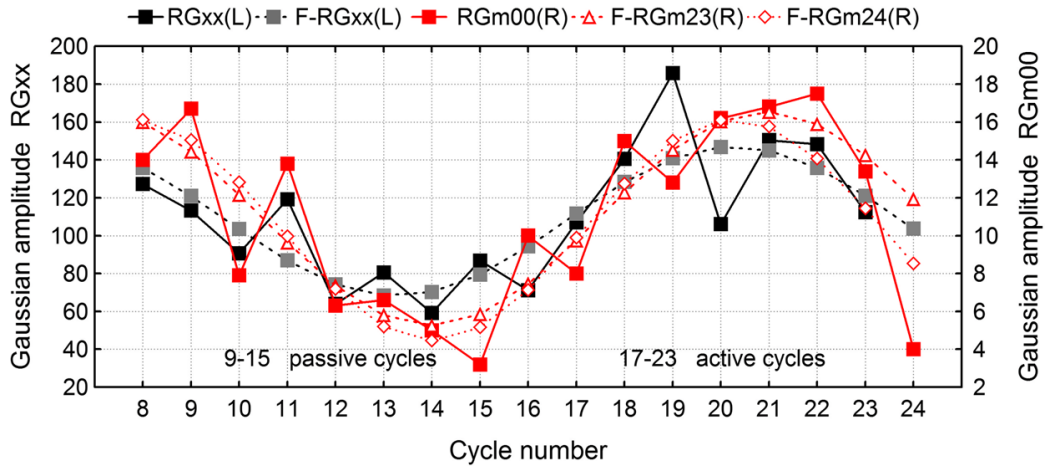

Figure 8 Scatterplot of the AI Gaussian maximum (RGxx, black) and PI Gaussian minimum (RGm00, red) amplitude. The best sinusoidal fit (F-RGxx) for the Gaussian maximum amplitude and the two best sinusoidal fits (with F-RGm24 and without F-RGm23 data for the PI 24) for the Gaussian minimum are shown.

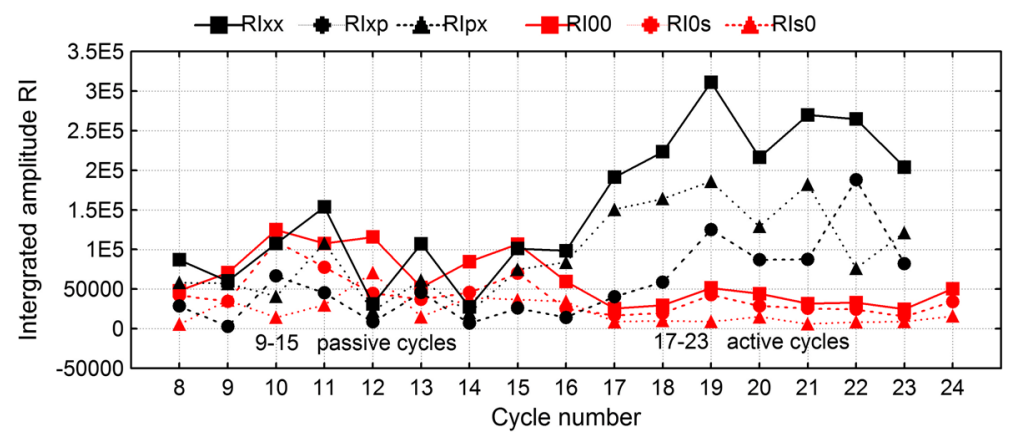

Figure 9 Similar to Figure 7, but in this case scatterplots of the integrated amplitude (RI) are shown.

and the AI integrated amplitude (RIxx in Figure 9) confirm this rule. The basic pattern of the curves in Figure 9 agrees with that in Figure 6.

The scatterplots of the AI peak, the mean and Gaussian amplitudes, and the PI Gaussian minimum amplitude suggest that these parameters can be fitted by sinusoids. The best fitted sinusoids, all in the form $y=a \cdot \sin (2 \pi \cdot(n-7) / T+\Phi)+b(n$ is the cycle number, and the other parameters are described in Table 4) are drawn in the corresponding figures.

All the best-fitted sinusoids indicate a period of about 14 solar 11-year cycles, which can be associated with the Gleissberg-cycle period (Gleissberg, 1939; Hathaway, Wilson, and Reichmann, 1999; Ogurtsov et al., 2002).

\section{The Relations Between the Time Distances and the Amplitudes}

\subsection{The Relations Between the Amplitude and the Period of Activity}

The first attempt to connect the amplitude of a cycle maximum with a selected time distance was made by Wolf (1861) who noticed that "greater activity on the Sun goes with shorter periods". This relationship is clearly seen especially when the integrated amplitude RIxx 
Table 4 Parameters of the sinusoids (Amplitude $=a \cdot \sin (2 \pi \cdot(n-7) / T+\Phi)+b$, where $n$ stands for the cycle number) that best fit the curves in Figures 6 to $8 . R^{2}$ (the coefficient of the determination) indicates how well data points fit a curve.

\begin{tabular}{llccccc}
\hline Amplitude & Symbol & \multicolumn{1}{c}{$T$, the period } & $\Phi$ & $b$ & $R^{2}$ \\
\hline RPxx & F-RPxx in Figure 6 & $41 \pm 20$ & $13.6 \pm 2.7$ & $1.8 \pm 0.9$ & $250 \pm 14$ & 0.27 \\
RMxx & F-RMxx in Figure 7 & $25 \pm 6$ & $12.0 \pm 1.2$ & $1.0 \pm 0.6$ & $91 \pm 5$ & 0.57 \\
RGxx & F-RGxx in Figure 8 & $40 \pm 8$ & $14.0 \pm 1.3$ & $1.9 \pm 0.4$ & $108 \pm 6$ & 0.65 \\
RGm00 & F-RGm23 in Figure 8 & $5.6 \pm 0.9$ & $13.9 \pm 1.2$ & $1.6 \pm 0.4$ & $11 \pm 0.7$ & 0.77 \\
RGm00 & F-RGm24 in Figure 8 & $5.8 \pm 1.0$ & $12.5 \pm 0.9$ & $1.2 \pm 0.4$ & $10 \pm 0.7$ & 0.71 \\
RGxx/RPxx & in Figure 8 in ZN & $0.086 \pm 0.008$ & $14.0 \pm 0.6$ & $1.9 \pm 0.2$ & $0.42 \pm 0.006$ & 0.90 \\
\hline
\end{tabular}

${ }^{1}$ Zięba and Nieckarz (2012).
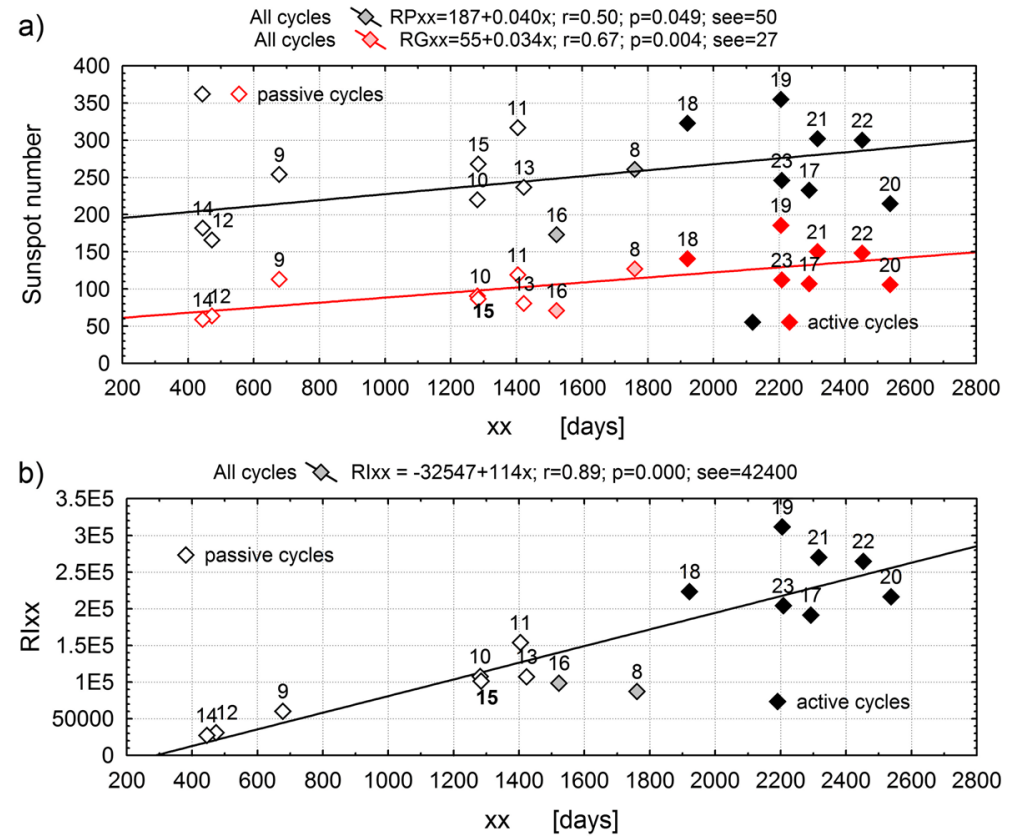

Figure 10 (a) Scatterplot of the peak (RPxx) and the Gaussian maximum (RGxx) versus lengths ' $x x^{\prime}$ ' for the indicated active intervals. (b) Scatterplot of the integrated amplitude (RIXx) versus ' $\mathrm{xx}$ '. The equation of the best linear fit and statistical parameters $r, p$, and the standard error of the estimation (see) are also given.

is taken as the measure of the solar activity. Figure 10 depicts the scatterplots of the peak maximum (RPxx), the Gaussian maximum (RGxx), and the integrated amplitude versus the active interval length ('xx').

Although these relationships have not directly a predictive value, 'xx' must be known from other relationships, they indicate a clear separation between passive and active cycles. The scatterplots in Figures 10(a) and (b) suggest high correlations among the three considered measures of the AI amplitude. The highest correlation coefficient, which equals 0.90 , is between the peak (RPxx) and the Gaussian maximum (RGxx) (Figure 11). 


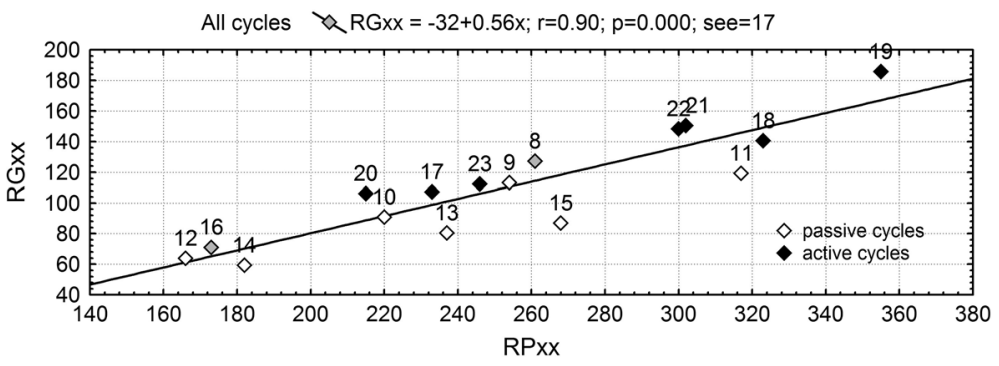

Figure 11 Scatterplot of the Gaussian maximum (RGxx) versus the peak maximum (RPxx) for the indicated AI. The equation of the best linear fit and statistical parameters $r, p$, and the standard error of the estimation (see) are also given.

\subsection{Waldmeier Effect}

The Waldmeier Effect (Waldmeier, 1935, 1939) is one of the widely quoted relationships in which the rise time elapsed between minimum and maximum of a cycle is inversely correlated with the cycle amplitude. The effect was analyzed using various monthly averages smoothed data sets such as: international (Wolf) and group sunspot numbers (Hathaway, Wilson, and Reichmann, 2002), Wolf sunspot numbers and sunspot area (Dikpati, Gilman, and de Toma, 2008), Wolf sunspot numbers, group sunspot numbers, sunspot area and 10.7 $\mathrm{cm}$ radio flux (Karak and Choudhuri, 2011). Among the time distances defined using the LSS location, the time distances 's0' and 'sp' are the closest to the most commonly used definition of the rise time when the cycle maximum has occurred. The correlation coefficients between 'sp' and the discussed amplitudes RPxx, RGxx and RIxx are not significant (they are about -0.23 ), while those between 's0' and these amplitudes are $-0.62,-0.76$, and -0.79 , respectively. All these values are significant with $p$-values smaller than 0.011 .

Beside the study of the classical Waldmeier effect, some authors have tried to find correlations showing that stronger cycles tend to rise faster (Lantos, 2000; Cameron and Schüssler, 2008; Kane, 2008; Podladchikova, Lefebvre, and van der Linden, 2008; Karak and Choudhuri, 2011). There are no simple observables to measure the growth and decay rates of solar activity. Various authors proposed different approaches to define a parameter measuring a growth rate of solar activity. All these authors used monthly averages smoothed data, but took differently defined time distance values (Lantos, 2000; Karak and Choudhuri, 2011) or sunspot numbers difference (Cameron and Schüssler, 2008) between two separated months selected during the cycle's ascending phase for their growth rate definition. Because many of the above-mentioned articles show that growth and decay rates can be treated as a precursor quantity, we examine for this purpose two values, RP0s/0s and RPs0/s0, formed by such observables as the segments ' $\mathrm{s} 0$ ' and 's0' as well as the maximal sunspot number in these segments RP0s and RPs0. We call these ratios declining and rising indices.

Figures 13 show the best linear relations for the amplitudes versus RP0s/0s while Figures 14 correspond to the same amplitudes versus RPs0/s0. Comparing the curves presented in both of these figures, we can see that the scatter of points representing the passive cycles in Figures 13 distort the relations. Similarly, the scatter of points representing the active cycles in Figures 14 decrease the correlations. Scatterplots drawn separately for the passive and active cycles are depicted in Figure 15. The relations present in Figure 12 can be seen as an equivalent of Waldmeier effect WE1, while those in Figure 14 can be seen as the Wald- 

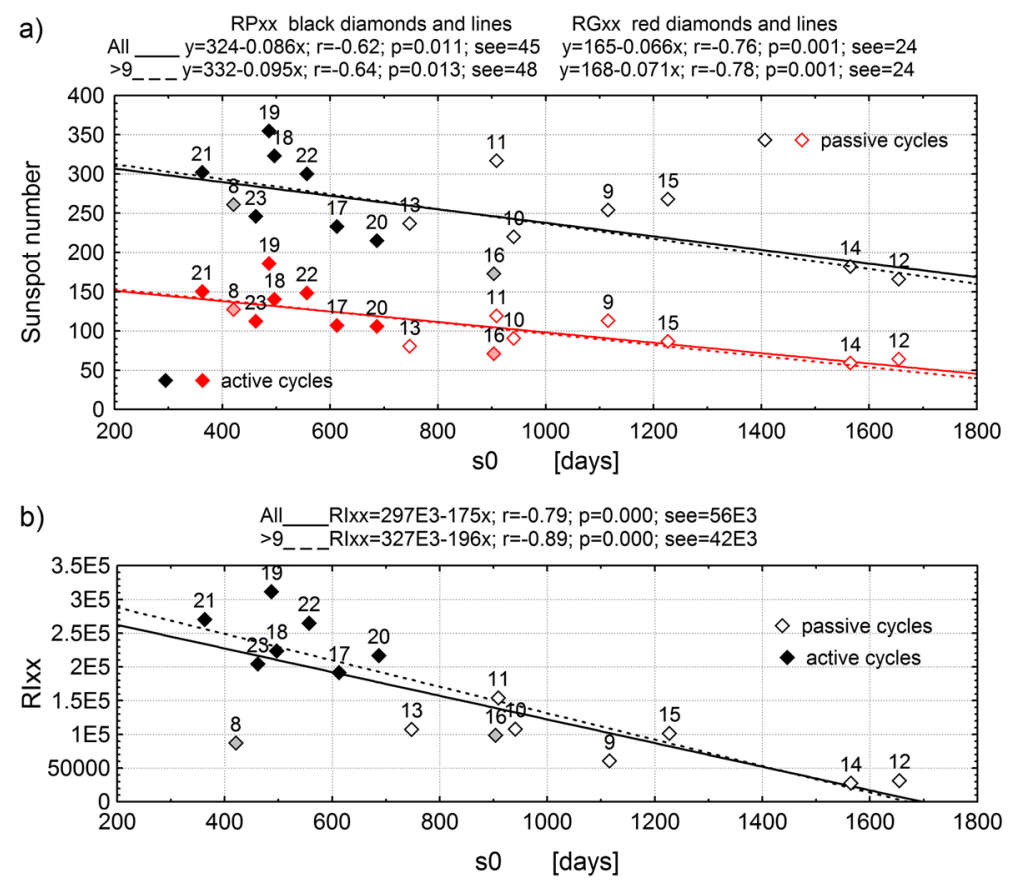

Figure 12 (a) Scatterplot of the peak (RPxx) and the Gaussian maximum (RGxx), and (b) scatterplot of the integrated amplitude (RIxx) versus lengths of the rising segment of the related passive intervals 's0' for the indicated AI. The equation of the best linear fit and statistical parameters $r, p$, and the standard error of the estimation (see) are also given. 'All' denotes Cycles 8-23, while '> 9' denotes Cycles 10-23.

meier effect WE2, in accordance with the distinction introduced by Karak and Choudhuri (2011).

Although both of the specified types of cycles are small samples, the correlations between the peak maximum and the RPs0/s0 for the passive cycles and between RP0s/0s for the active ones are high and statistically significant. Taking into account both of these indices and the other observables describing passive intervals, we have constructed, separately for the passive and active cycles, the best multiple-linear regression models for the maximal sunspot number RPxx and RGxx of the studied cycles. In Table 5, we present the obtained coefficients of the models together with the statistical significance of the estimated parameters and $R^{2}$ value, which checks the goodness of the fit.

\section{Predictions for Cycle 24}

In this section we address the prediction of the timings and the amplitude of the ongoing sunspot active interval for Cycle 24, using relations based on the description of the sunspot time series as a sequence of non-overlapping segments called passive and active intervals. Some relations inferred from the location of LSS with respect to the occurrences of the FSD and the former sunspot maximum were discussed in Zięba and Nieckarz (2012) for a prediction of the peak and the Gaussian-smoothed maximum sunspot number for the present cycle. 

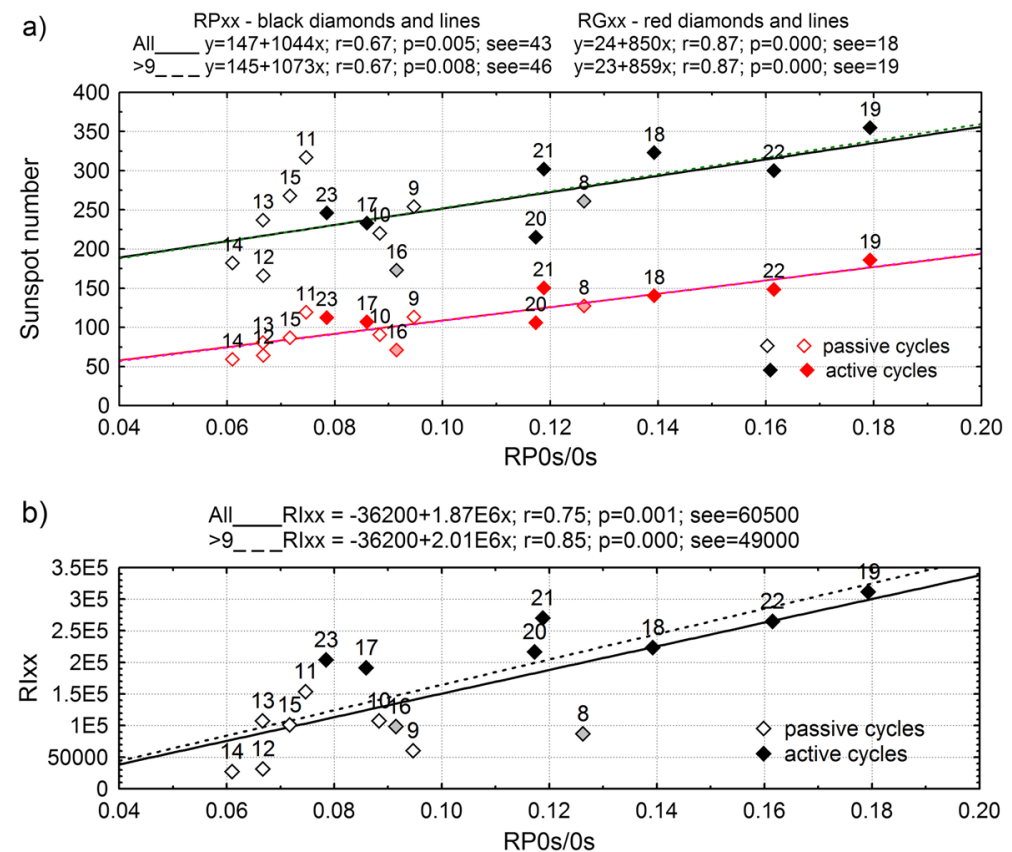

Figure 13 Similar to Figures 12(a) and (b), but in this case the scatterplots correspond to the amplitudes versus $\mathrm{RP} 0 \mathrm{~s} / 0 \mathrm{~s}$.
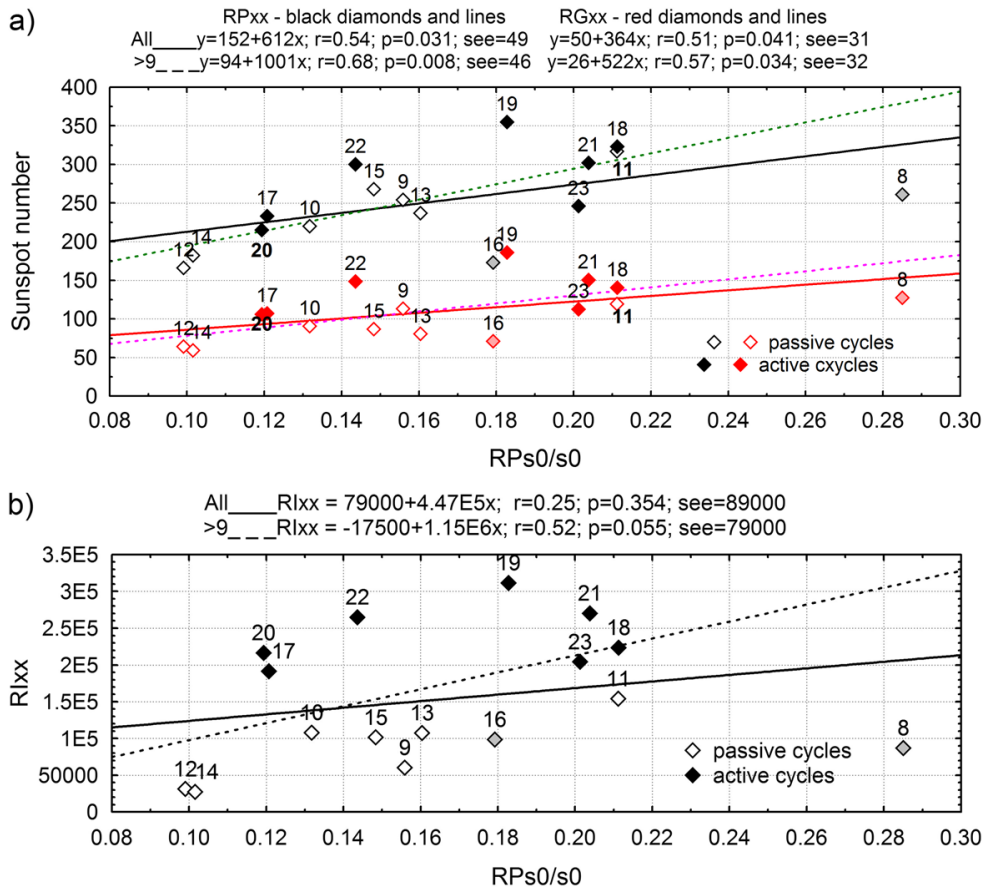

Figure 14 Similar to Figures 13(a) and (b), but in this case the scatterplots correspond to the amplitudes versus $\mathrm{RPs} 0 / \mathrm{s} 0$. 
Figure 15 Scatterplots of the peak and the Gaussian maximum amplitudes drawn for the passive (a) and active (b) cycles versus $\mathrm{RPs} 0 / \mathrm{s} 0$ and $\mathrm{RP} 0 \mathrm{~s} / 0 \mathrm{~s}$, respectively.
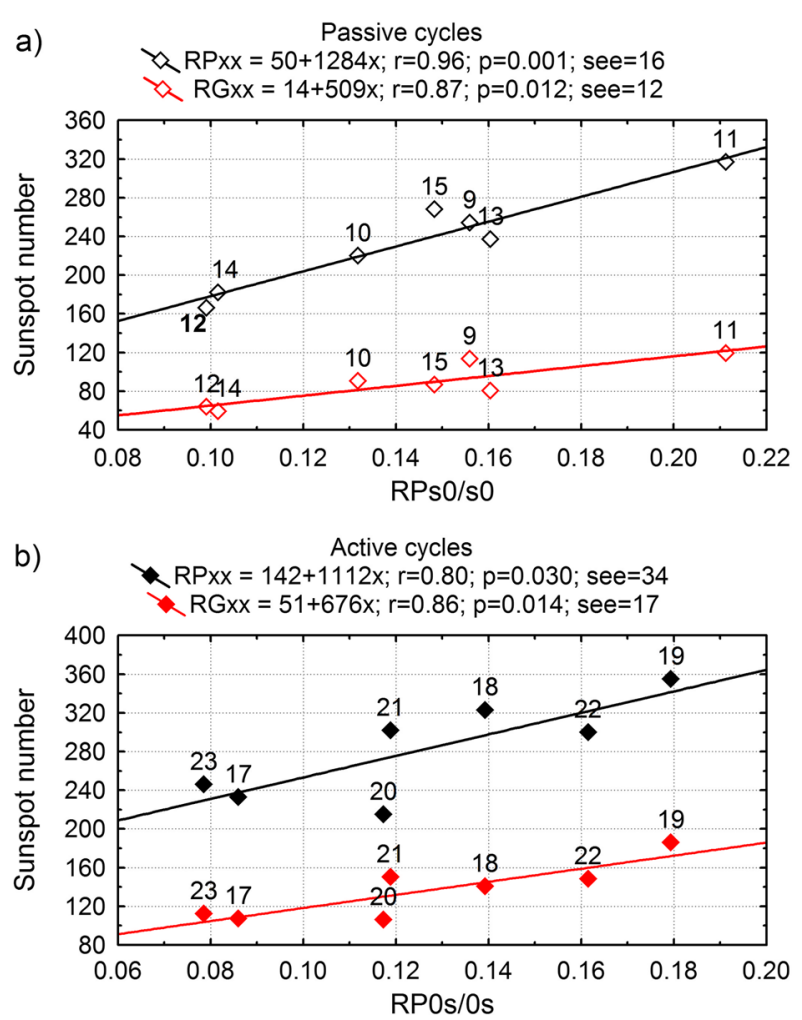

To assess the length of the ongoing active interval we have examined how the PI lengths '00', '0s' and 's0' affected the length ' $\mathrm{xx}$ ' of the subsequent active interval. The highest correlation coefficient was found between ' $\mathrm{xx}$ ' and ' $\mathrm{s} 0$ '. Figure 16 presents this relation and indicates a clear concentration of active cycles near the coordinates $(524,2276)$. It is evident, from the curves for the passive and active cycles in Figure 17, that the lengths of the active cycles do not correlate with the 's0' lengths, while the lengths of the passive cycles do.

The position of the peak maximum can also be predicted from the relations between the time distance '0p' and the PI lengths '00' as well as '0s' (Figure 18). The correlations of these parameters with the position of the Gaussian maximum ' $0 \mathrm{~g}$ ' are higher than those with ' 0 p' and are equal to 0.97 and 0.96 , respectively.

Assuming that the ongoing Cycle 24 is a passive one, we calculated (Table 6) the peak and Gaussian maxima and other parameters for this cycle, using the models presented in Table 5 and some other relations given above.

The number of days, which elapsed from the last spotless day of the PI of Cycle 24, is 809 days counted from 31 October 2013 (the day on which this article was submitted). According to the relation presented in Figure 17, we assume that the end of the maximum phase of Cycle 24 (i.e. the active interval) can occur in the second half of 2015. The daily maximal ISN was 136 on 21 October 2011, but on 16 May 2013 this number was 135. Our predictions for the day of the peak maximum do not exclude that it can still happen, but the maximum value of the sunspot number is smaller than the one predicted by us (198) up to now. Our prediction for the Gaussian maximum is that it will happen around 100 days after the peak maximum. 


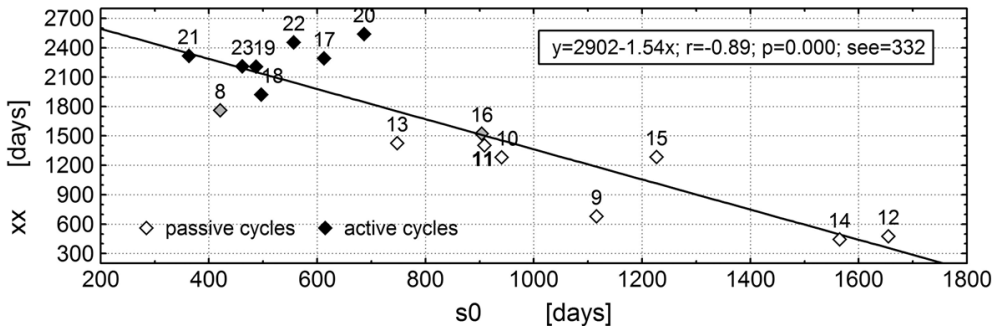

Figure 16 Scatterplot of the active interval lengths ' $x x$ ' versus lengths of the PI rising segments 's0'. The equation of the best linear fit and statistical parameters $r, p$, and the standard error of the estimation (see) are also given.

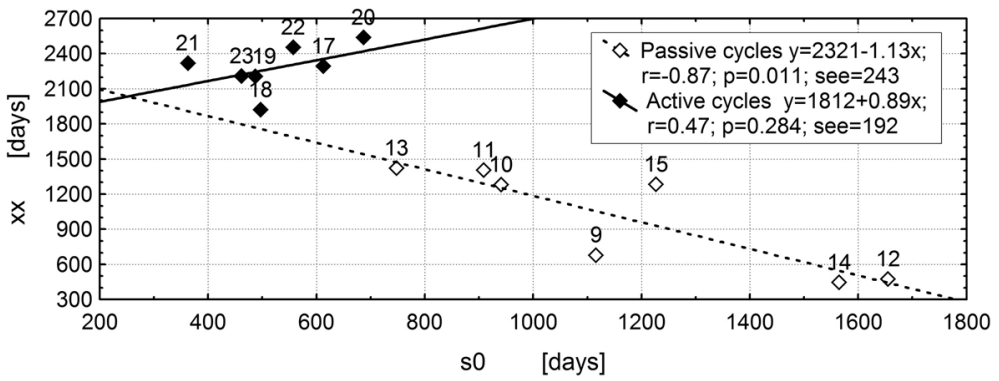

Figure 17 Similar to Figure 16, but separating the passive and active cycles.
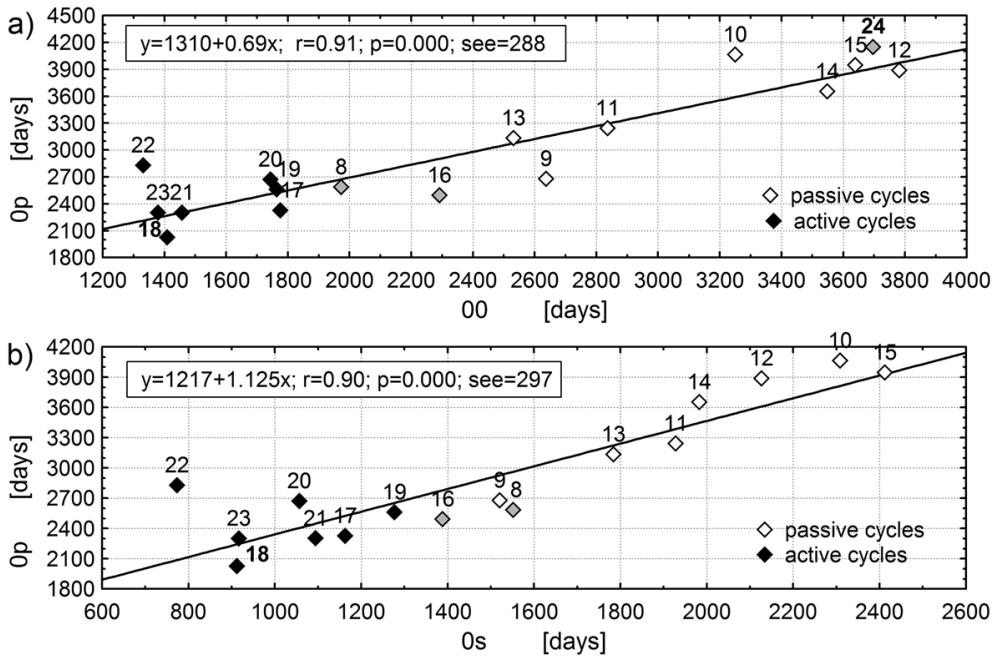

Figure 18 In (a) the position of the peak maximum with respect to the FSD of the passive interval ' 0 p' versus the length of the PI '00' and in (b) versus the length of the PI declining segment ' 0 s'. The equation of the best linear fit and statistical parameters $r, p$, and the standard error of the estimation (see) are also given. 
Table 5 The parameters of the multi-linear regression models for the prediction of the maximal peak (RPxx) and Gaussian amplitude (RGxx) of the studied passive or active cycles using the following predictors (variables): RP0s/0s, the declining index, RPs0/s0, the rising index, nlss, the number of the spotless days in the LSS, n00, the number of the spotless days in the passive interval.

\begin{tabular}{|c|c|c|c|c|c|c|}
\hline \multicolumn{6}{|c|}{ Model: $\mathrm{RPxx}=a+\mathrm{b} 1 \cdot \mathrm{RP} 0 \mathrm{~s} / 0 \mathrm{~s}+\mathrm{b} 2 \cdot \mathrm{RPs} 0 / \mathrm{s} 0+\mathrm{b} 3 \cdot \mathrm{nlss}$} & \multirow{2}{*}{$\begin{array}{l}\text { Analysis of variance for } \\
{ }^{3} \text { Regression }\end{array}$} \\
\hline \multirow[t]{2}{*}{ Variable } & Coefficient & Value & ${ }^{1}$ Std. Error & & alue & \\
\hline & $a$ & -50.306 & 24.9104 & \multicolumn{2}{|c|}{0.13672} & \multirow{4}{*}{$\begin{array}{l}\mathrm{SSM}=15834.29 \mathrm{SSE}=138.57 \\
F=114.27 p \text {-value }=0.00137 \\
R^{2}=0.9956 \\
\text { Adjusted } R^{2}=0.9826 \text { see }=6.8\end{array}$} \\
\hline \multirow{3}{*}{$\begin{array}{l}\mathrm{RP0s} / 0 \mathrm{~s} \\
\mathrm{RPs} 0 / \mathrm{s} 0 \\
\text { nlss }\end{array}$} & b1 & 729.391 & 254.7788 & \multicolumn{2}{|c|}{0.06443} & \\
\hline & $\mathrm{b} 2$ & 1380.260 & 79.6949 & \multicolumn{2}{|c|}{0.00042} & \\
\hline & b3 & 0.637 & 0.1316 & \multicolumn{2}{|c|}{0.01685} & \\
\hline \multicolumn{6}{|c|}{ Model: RGxx $=a+\mathrm{b} 1 \cdot \mathrm{RP} 0 \mathrm{~s} / 0 \mathrm{~s}+\mathrm{b} 2 \cdot \mathrm{RPs} 0 / \mathrm{s} 0$} & \multirow{2}{*}{$\begin{array}{l}\text { Analysis of variance for } \\
\text { Regression }\end{array}$} \\
\hline \multirow[t]{2}{*}{ Variable } & Coefficient & Value & \multicolumn{3}{|c|}{ Std. Error } & \\
\hline & \multicolumn{2}{|c|}{$a \quad-41.096$} & \multicolumn{2}{|c|}{12.4554} & 0.02995 & \multirow{3}{*}{$\begin{array}{l}\mathrm{SSM}=2993.77 \mathrm{SSE}=89.04 \\
F=67.24 p \text {-value }=0.00083 \\
R^{2}=0.9711 \\
\text { Adjusted } R^{2}=0.95667 \text { see }=4.7\end{array}$} \\
\hline $\mathrm{RPOs} / 0 \mathrm{~s}$ & b1 & 909.152 & 164.3 & & 0.00521 & \\
\hline $\mathrm{RPs} 0 / \mathrm{s} 0$ & $\mathrm{~b} 2$ & 421.323 & 52.4 & & 0.0013 & \\
\hline \multicolumn{7}{|c|}{ Active cycle } \\
\hline \multicolumn{6}{|c|}{ Model: $\mathrm{RPxx}=a+\mathrm{b} 1 \cdot \mathrm{RP} 0 \mathrm{~s} / 0 \mathrm{~s}+\mathrm{b} 2 \cdot \mathrm{RPs} 0 / \mathrm{s} 0+\mathrm{b} 3 \cdot \mathrm{n} 00$} & \multirow{2}{*}{$\begin{array}{l}\text { Analysis of variance for } \\
\text { Regression }\end{array}$} \\
\hline \multirow[t]{2}{*}{ Variable } & Coefficient & Value & Std. Error & \multicolumn{2}{|c|}{$p$-value } & \\
\hline & $a$ & -22.716 & 27.4782 & 0.4 & & \multirow{4}{*}{$\begin{array}{l}\mathrm{SSM}=15634.5 \mathrm{SSE}=285.5 \\
F=54.75 p \text {-value }=0.004056 \\
R^{2}=0.9910 \\
\text { Adjusted } R^{2}=0.9641 \text { see }=9.8\end{array}$} \\
\hline \multirow{3}{*}{$\begin{array}{l}\mathrm{RP} 0 \mathrm{~s} / 0 \mathrm{~s} \\
\mathrm{RPs} 0 / \mathrm{s} 0 \\
\mathrm{n} 00\end{array}$} & b1 & 1074.876 & 108.6869 & 0.0 & & \\
\hline & b2 & 771.194 & 103.9258 & 0.0 & & \\
\hline & b3 & 0.116 & 0.0340 & 0.0 & & \\
\hline \multicolumn{6}{|c|}{ Model: $\mathrm{RGxx}=a+\mathrm{b} 1 \cdot \mathrm{RP} 0 \mathrm{~s} / 0 \mathrm{~s}+\mathrm{b} 2 \cdot \mathrm{RPs} 0 / \mathrm{s} 0+\mathrm{b} 3 \cdot \mathrm{n} 00$} & \multirow{2}{*}{$\begin{array}{l}\text { Analysis of variance for } \\
\text { Regression }\end{array}$} \\
\hline \multirow[t]{2}{*}{ Variable } & Coefficient & Value & Std. Error & \multicolumn{2}{|c|}{$p$-value } & \\
\hline & $a$ & -25.040 & 30.1489 & \multicolumn{2}{|c|}{0.46714} & \multirow{4}{*}{$\begin{array}{l}\mathrm{SSM}=4807.0 \mathrm{SSE}=343.7 \\
F=13.98 p \text {-value }=0.02868 \\
R^{2}=0.9333 \\
\text { Adjusted } R^{2}=0.8665 \text { see }=10.7\end{array}$} \\
\hline $\mathrm{RPOs} / 0 \mathrm{~s}$ & b1 & 669.175 & 119.2504 & \multicolumn{2}{|c|}{0.01119} & \\
\hline $\mathrm{RPs} 0 / \mathrm{s} 0$ & $\mathrm{~b} 2$ & 321.174 & 114.0266 & \multicolumn{2}{|c|}{0.06692} & \\
\hline n00 & b3 & 0.0662 & 0.0373 & \multicolumn{2}{|c|}{0.17409} & \\
\hline
\end{tabular}

${ }^{1}$ Std. Error, estimates how much a coefficient is likely to vary, similar to the standard error of the mean (sem).

$2 p$-value the probability of a test statistic used for verifying the null hypothesis.

${ }^{3} \mathrm{SSM}$, the model sum of squares, it is the sum of squared deviations of the model predicted values from the mean of the observed values, SSE, residuals sum of squares, $F$, F-test statistic, used in testing the model validation, $R^{2}$, the coefficient of determination, indicates how well data points fits a statistical model. An $R^{2}$ of 1 indicates that the model perfectly fits the data, Adjusted $R^{2}$ is a modification of $R^{2}$ that adjusts for the number of input variable in a model relative to the number of data points, 'see' is the standard error of the estimation.

\section{Summary and Discussion}

Taking into account spotless days, we have represented the ISN time series as the sequence of precisely defined consecutive time intervals, called passive and active intervals, according 
Table 6 Some predictions for the AI in Cycle 24 derived from the relations found from previous cycles.

\begin{tabular}{|c|c|c|c|c|c|c|c|c|}
\hline \multicolumn{2}{|l|}{ Variables } & \multirow[t]{2}{*}{$r$} & \multirow[t]{2}{*}{ Fig. } & \multicolumn{2}{|c|}{$\begin{array}{l}\text { Equation } \\
y=a+b x\end{array}$} & \multirow[t]{2}{*}{ See } & \multirow[t]{2}{*}{$y$} & \multirow[t]{2}{*}{$\begin{array}{l}\text { Prediction for the } \\
\text { AI of Cycle } 24\end{array}$} \\
\hline$x$ & $y$ & & & $a$ & $b$ & & & \\
\hline $\mathrm{s} 0=727$ & $\mathrm{XX}$ & -0.89 & 17 & 2321 & -1.13 & 243 & 1499 & $\begin{array}{l}{ }^{1} \text { FSD } 72217 \\
\text { Sep } 2015\end{array}$ \\
\hline \multirow[t]{2}{*}{$00=2757$} & $0 p$ & 0.91 & $18 \mathrm{a}$ & 1310 & 0.69 & 248 & 3212 & $\begin{array}{l}{ }^{2} \text { DPx } 71174 \\
\text { Nov } 2012\end{array}$ \\
\hline & $0 \mathrm{~g}$ & 0.97 & - & 1414 & 0.69 & 148 & 3316 & $\begin{array}{l}2 \text { DGx } 71278 \\
\text { Feb } 2013\end{array}$ \\
\hline \multirow[t]{2}{*}{$0 s=2029$} & $0 p$ & 0.90 & $18 b$ & 1218 & 1.12 & 297 & 3490 & $\begin{array}{l}\text { DPx } 71452 \\
\text { Aug } 2013\end{array}$ \\
\hline & $0 \mathrm{~g}$ & 0.96 & - & 1327 & 1.12 & 170 & 3599 & $\begin{array}{l}\text { DGx } 71561 \\
\text { Dec } 2013\end{array}$ \\
\hline $\mathrm{RP} 0 \mathrm{~s} / 0 \mathrm{~s}=0.0527$ & RPxx & $\begin{array}{l}R^{2} \\
0.983\end{array}$ & \multirow{2}{*}{\multicolumn{3}{|c|}{$\begin{array}{l}\text { See Table } 5 \text { for the } \\
\text { parameters of the linear } \\
\text { regression. }\end{array}$}} & 7 & 198 & $\begin{array}{l}95 \% \text { CI } \\
175-222\end{array}$ \\
\hline $\begin{array}{l}\mathrm{RPs} 0 / \mathrm{s} 0=0.1375 \\
\text { nlss }=32\end{array}$ & RGxx & $\begin{array}{l}R^{2} \\
0.956\end{array}$ & & & & 5 & 64 & $54-76$ \\
\hline
\end{tabular}

${ }^{1}$ FSD, the first spotless day of the PI of Cycle 25 at the end if of the AI of Cycle 24.

2 DPx, DGx, the day of the peak and Gaussian maximum, respectively.

to whether they contain minimal or maximal daily sunspot numbers. Active intervals correspond to a phase of maximal sunspot numbers (both the peak and the Gaussian maximum are inside an $\mathrm{AI}$ ), but passive intervals are extended beyond a classically understood minimum phase of the solar cycle and include the declining phase of the previous maximum. The properties of each passive interval, such as characteristic lengths, numbers of spotless days, declining, and rising indices are predictors of the length and amplitudes of the approaching active interval.

We have found clear differences between properties of two types of solar cycles (see Table 3) referred to as the passive (Cycles 9-15) and active (Cycles 17-23) cycles. Each group contains seven cycles in a row. According to the Hathaway division of cycles into small, medium and large (Hathaway, 2010, 2011), the passive cycles are small and medium ones, while the active cycles are large and medium. The distribution of days with spots having a relatively high sunspot number is different in the both types of cycles (see Figure 5). All the three discussed cycle amplitudes (the peak, the Gaussian, and the integrated one) show a variation with time that can be fitted using sinusoidal functions. The periods of these functions (see Table 4) change from 12 to 14 solar 11-year periods. These facts indicate that the occurrence of passive and active cycles can be connected with a higher mode of the Gleisberg cycle (Gleissberg, 1939; Ogurtsov et al., 2002).

Analyzing the influence of different properties of the passive intervals on the length and amplitudes of the related active intervals we have shown that solar activity in the PI declining-segment (a decay phase of the previous cycle) has a greater impact on the strength of the approaching maximum in the case of the active cycles, while activity in the PI risingsegments in the case of the passive cycles. To circumscribe solar activity in the PI segments we have introduced indices defined as a ratio of the maximal sunspot number recorded during the declining or the rising PI segment to the length of this segment (RP0s/0s and RPs0/s0, respectively). We have found multi-linear relations (see Table 5) predicting the strength of the approaching maximum using the introduced indices. The linear version of these models 


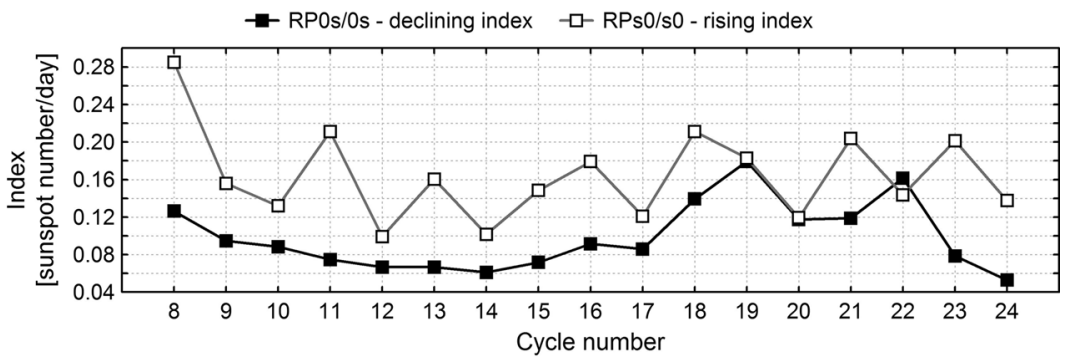

Figure 19 Values of the declining and rising index calculated for the subsequent passive intervals (epochs of solar minimum previous to the maxima indicated by the cycle number).

(see Figure 15) can be considered as the Waldmeier effect WE2, while the relation seen in Figure 12 can be seen as the Waldmeier effect WE1, in accordance with the distinction introduced by Karak and Choudhuri (Karak and Choudhuri, 2011).

The variations of these indices, which are the main predictors of the multi-linear models, with solar cycles are presented in Figure 19. The declining index (RP0s/0s) is small and practically constant for the passive cycles and rises to a statistically significantly higher values for the active cycles. The opposite is observed for the rising index (RPs0/s0). Its values fluctuate around 0.16 in both groups.

The main questions resulting from our analysis based on the daily sunspot time series can be formulated as follows:

i) What is the cause of the apparent difference between passive and active cycles? We can answer that it can be either the specific cycle phase division based on the spotless days or that there is a key physical reason that underlies the different observed distribution of days with a relatively high sunspot number for this group of cycles. The fact that the passive and active cycles occur in a row, and in a number which corresponds to the Gleisberg cycle, indicates a possible underlying physical cause.

ii) The cycle overlapping effect revealed by Cameron and Schüssler $(2007,2008)$ can explain the high fluctuation of the sunspot numbers of the passive cycles during their passive intervals (a relatively large overlapping) as well as the marked reduction of this fluctuation in the case of the active cycles (a small overlapping). This is also confirmed by the large number of spotless days during the passive cycles because their long passive intervals lead to more days without activity even if some days have relatively large sunspot numbers.

However, we cannot say whether changes of the declining index (see Figure 19) are related only to the overlapping of cycles and the Waldmeier effect (Cameron and Schüssler, 2007), or whether they are connected also with the meridional plasma flow variations considered by Nandy, Muñoz-Jaramillo, and Martens (2011). Similarly, it is difficult to assume without further study that one of the various possible mechanisms, i.e. the evolution of the polar field around solar minimum, could explain the observed fluctuations of our rising index (Cameron et al., 2010, 2013; Dasi-Espuig et al., 2010; Jiang et al., 2010; Cameron and Schüssler, 2012).

Svalgaard and Kamide (2013) have shown that the polar fields play a crucial role in the solar cycle. Because of solar-cycle $\mathrm{N}-\mathrm{S}$ asymmetries, polar-field reversals do not occur at the same times in both hemispheres (Norton and Gallagher, 2010; Muñoz-Jaramillo et al., 2013b, 2013a; Zhao, Landi, and Gibson, 2013), and some differences among various passive intervals may be expected. The long-term persistence of a phase leading in one of the 
hemispheres (Zolotova et al., 2009) can explain a repetition of some noticed properties of passive and active intervals.

The focus of this article is to analyze the daily sunspot time series using the days for which timing and sunspot number are precisely defined, and we have done so. We have, however, found some other facts and characteristics that will be the object of further analysis.

Acknowledgements We thank the anonymous referee for constructive comments and suggestions, which much improved the original version of the manuscript.

Open Access This article is distributed under the terms of the Creative Commons Attribution License which permits any use, distribution, and reproduction in any medium, provided the original author(s) and the source are credited.

\section{References}

Cameron, R.H., Schüssler, M.: 2007, Astrophys. J. 659, 801.

Cameron, R., Schüssler, M.: 2008, Astrophys. J. 685, 1291.

Cameron, R.H., Schüssler, M.: 2012, Astron. Astrophys. 548, A57.

Cameron, R.H., Das-Espuig, M., Jiang, J., Işik, E., Schmitt, D., Schüssler, M.: 2013, Astron. Astrophys. 557, A141.

Cameron, R.H., Jiang, J., Schmitt, D., Schüssler, M.: 2010, Astrophys. J. 719, 264.

Clette, F., Berghmans, D., Vanlommel, P., Van der Linden, R.A.M., Koeckelenbergh, A., Wauters, L.: 2007, Adv. Space Res. 40, 919.

Dasi-Espuig, M., Solanki, S.K., Krivova, N.A., Cameron, R.H., Peñuela, T.: 2010, Astron. Astrophys. 518, A97.

Dikpati, M., Gilman, P.A., de Toma, G.: 2008, Astrophys. J. 673, L99.

Gleissberg, W.: 1939, Observatory 62, 158.

Gnevyshev, M.N., Ohl, A.I.: 1948, Astron. Zh. 25, 8.

Harvey, K.L., White, O.R.: 1999, J. Geophys. Res. 104, 19759.

Hathaway, D.H.: 2010, Living Rev. Solar Phys. 7, 1.

Hathaway, D.H.: 2011, Solar Phys. 273, 221.

Hathaway, D.H., Wilson, R.M., Reichmann, E.J.: 1999, J. Geophys. Res. 104, 22375.

Hathaway, D.H., Wilson, R.M., Reichmann, E.J.: 2002, Solar Phys. 211, 357.

Jiang, J., Işik, E., Cameron, R.H., Schmitt, D., Schüssler, M.: 2010, Astrophys. J. 717, 597.

Kane, R.P.: 2008, Solar Phys. 248, 203.

Karak, B.B., Choudhuri, A.R.: 2011, Mon. Not. Roy. Astron. Soc. 410, 1503.

Kopecky, M.: 1950, Bull. Astron. Inst. Czechoslov. 2, 14.

Lantos, P.: 2000, Solar Phys. 196, 221.

McKinnon, J.A.: 1987, UAG Repots UAG-95, National Geophysical Data Center, NOAA, Boulder, CO.

Muñoz-Jaramillo, A., Balmaceda, L.A., DeLuca, E.E.: 2013a, Phys. Rev. Lett. 11, 041106.

Muñoz-Jaramillo, A., Dasi-Espuig, M., Balmaceda, L.A., DeLuca, E.E.: 2013b, Astrophys. J. Lett. 767, L25.

Nandy, D., Muñoz-Jaramillo, A., Martens, P.C.H.: 2011, Nature 471, 80.

Norton, A.A., Gallagher, J.C.: 2010, Solar Phys. 261, 193.

Ogurtsov, M.G., Nagovitsyn, Y.A., Kocharov, G.E., Jungner, H.: 2002, Solar Phys. $211,371$.

Podladchikova, T., Lefebvre, B., van der Linden, R.: 2008, J. Atmos. Solar-Terr. Phys. 70, 277.

Svalgaard, L., Kamide, Y.: 2013, Astrophys. J. 763, 23.

Waldmeier, M.: 1935, Astron. Mitt. Eidgenöss. Sternwarte Zür. 14(133), 105.

Waldmeier, M.: 1939, Astron. Mitt. Eidgenöss. Sternwarte Zür. 14(138), 470.

Waldmeier, M.: 1961, The Sunspot-Activity in the Years 1610-1960, Schulthess, Zürich.

Wilson, R.M.: 1995, Solar Phys. 152, 197.

Wilson, R.M.: 1998, Solar Phys. 182, 217.

Wilson, R.M., Hathaway, D.H.: 2005, NASA Technical Report, NASA/TP-2005-213608.

Wilson, R.M., Hathaway, D.H.: 2006, NASA Technical Report, NASA/TP-2006-214601.

Wilson, R.M., Hathaway, D.H.: 2007, NASA Technical Report, NASA/TP-2007-215134.

Wolf, R.: 1861, Mon. Not. Roy. Astron. Soc. 21, 77.

Zhao, L., Landi, E., Gibson, S.E.: 2013, Astrophys. J. 773, 157.

Zięba, S., Masłowski, J., Michalec, A., Michałek, G., Kułak, A.: 2006, Astrophys. J. 653, 1517.

Zięba, S., Nieckarz, Z.: 2012, Solar Phys. 278, 457.

Zolotova, N.V., Ponyavin, D.I., Marwan, N., Kurths, J.: 2009, Astron. Astrophys. 503, 197. 